\title{
Ellipsoid Spectacle Comparison of Plusoptix, Retinomax and 2WIN Autorefractors
}

\author{
Robert W Arnold (ID) \\ Samuel J Martin ${ }^{2}$ \\ Joshua R Beveridge 3 \\ Andrew W Arnold ${ }^{4}$ \\ Stephanie L Arnold ${ }^{4}$ \\ Nathanael R Beveridge 5 \\ Kyle A Smith $\mathbb{D}^{1,6}$

\begin{abstract}
'Alaska Blind Child Discovery, Alaska AK, 99508, USA; ${ }^{2}$ Loma Linda Medical School, Loma Linda, CA, USA; ${ }^{3}$ ddaho College of Osteopathic Medicine, Boise, ID, USA; ${ }^{4}$ Ascension Genesys Residency Program, Grand Blanc, MI, USA; ${ }^{5}$ US Airforce Academy, Colorado Springs, CO, USA; ${ }^{6}$ Accurate Vision, Anchorage, AK, USA
\end{abstract} \\ Children's EYE \& Strabismus, Anchorage,
}

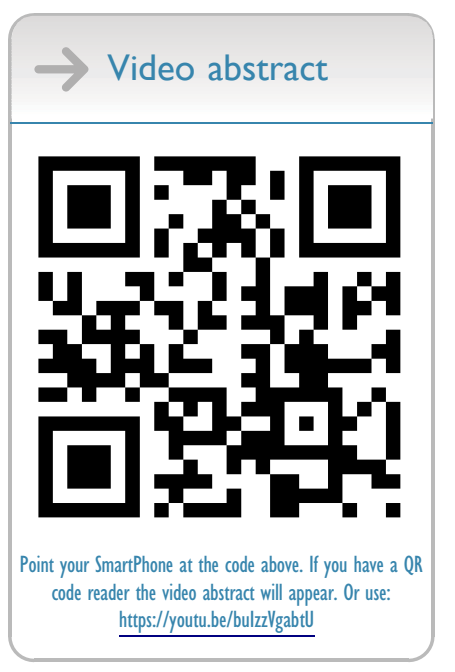

Correspondence: Robert W Arnold Alaska Children's EYE \& Strabismus, 3500 Latouche Street \#280, Anchorage, AK, 99508, USA

Tel + I(907)56I-1917

Fax + 9075635373

Email eyedoc@alaska.net
Background: Handheld devices can automatically give an estimate of refraction. The established method for refraction comparison using spherical equivalent (M) and J0, J45 vector transformations by Bland-Altman analysis is too complex for non-eye doctors involved with vision screening and remote vision clinics. Therefore, a simpler comparison technique was developed.

Methods: Based on the spectacle limit to resolve grade A $1 \log$ AAR, B $3 \log$ MAR and C 6 $\log$ MAR blur, J0, J45, and M are combined into the Alaska Blind Child Discovery (ABCD) composite ellipsoid GRADE system. Pediatric eye patients had confirmatory examination after dry refraction with three portable autorefractors: Plusoptix, 2WIN and Retinomax. The refractions were then compared using both Bland-Altman and ABCD composite. Performance to detect AAPOS amblyopia risk factors was also assessed.

Results: A total of 202 children, mean age seven years, $28 \%$ high spectacle need and $43 \%$ AAPOS 2013 amblyopia risk factors showed high correlation with cycloplegic refraction (intraclass correlation 0.49 to 0.90 ) for sphere, J0 and J45 spectacle components. Plusoptix had more $(10 \%)$ inconclusives due to patients out-of-range. The Retinomax was unable to screen some younger children and was less reliable for sphere but gave more precise astigmatism estimates. The proportion of autorefractions expected to give GRADE A/B highneed patients acuity improvement to $20 / 40$ would be $41 \%$ for Plusoptix, $39 \%$ for $2 \mathrm{WIN}$ and $65 \%$ for Retinomax. Sensitivity/specificity for amblyopia risk factor detection was $80 \% / 83 \%$ for Plusoptix, $72 \% / 88 \%$ for 2 WIN and $84 \% / 73 \%$ for Retinomax.

Conclusion: The simplified spectacle comparison resembled Bland-Altman and could assist lay vision screeners and non-eye doctors attempting remote spectacle donation worldwide.

Keywords: spectacles, remote dispensing, autorefractors, validation

\section{Introduction}

Dispensing correct spectacles is one of the most important aspects of remote vision clinics $^{1}$ and it is also a critical component in the effective treatment of amblyopia. ${ }^{2}$ At times, spherocylinder spectacle prescriptions should be compared.

A standard method to compare spectacle prescriptions is to break the spherocylinder format into spherical equivalent, and vector transformed astigmatism components $\mathrm{J} 0$ and $\mathrm{J} 45 .^{3}$ Each of these linear continuous variables can then be correlated and subjected to Bland-Altman analysis. Bland-Altman is related to correlation and, in practice should be preceded by a correlation calculation. In Bland-Altman, wellmatching data form a horizontal rather than oblique configuration, and the quality of matches is identified by ranges of disparity on the vertical axis. Although these vector spectacle comparison techniques are the current preferred standard, these mathematical 
constructs are complicated and confusing for lay vision screeners or remote health clinic workers responsible for handing out glasses.

One indication for comparison of refractions is the performance of instruments designed to estimate spectacle refractions by various optical techniques. In pediatric ophthalmology, amblyopia vision screening and remote rural eye clinics, portable, battery-powered handheld devices estimate spectacle sphere, cylinder and axis for right and left eyes. In children and adults, it is important to know the accuracy and precision of such interpretations by each type of device.

We sought a simpler and clinically pertinent method for spectacle comparison and therefore combined the J0 and J45 cylinder components into $\mathrm{x}, \mathrm{y}$ coordinates. Subjects demonstrated best corrected normal visual acuity before a determination of the limits of spherocylinder with isospherical equivalent that allowed visual acuity at uniform levels of blur. We selected $1 \operatorname{logMAR}$ blur defined as A-grade blur, 3 $\log$ MAR as B-grade blur and $6 \log$ MAR defined as C-grade blur. The vector transform from the limits of graded blur formed concentric ovals or circles on a J0 vs J45 coordinate. ${ }^{4}$ Our original effort also calculated an age-related grade for spherical equivalent recognizing that younger subjects are capable of resolving clear images with more over-minus than older patients who have less accommodation.

In this project, the Alaska Blind Child Discovery (ABCD) presents an additional simplifying step called the "ABCD composite" that combines a simplified cylinder J0 vs J45 component with the age-related spherical equivalent grade resulting in a single measure of combined spherocylinder that we call the "ellipsoid" grade comparison. Then we apply the simplified ellipsoid grade for three portable autorefractors compared to best, refined retinoscopy directed refraction. Our standard comparison is Bland-Altman analysis after intraclass correlation.

\section{Methods}

This validation study, an evaluation of a clinical test, has institutional review from Providence Alaska Medical Center and complies with the Health Insurance Portability and Accessibility Act and the Declaration of Helsinki. Parents and older subjects provided written informed consent.

The first aspect was enlisting the aid of a $\mathrm{PhD}$ candidate mathematician (NRB) to develop a three-dimensional mathematical construct of the spectacle spherocylinder refraction resembling an ellipsoid. The second aspect was applying the spectacle match grade ellipsoid to three remote autorefractors with intraclass correlation and Bland-Altman for comparison. The third was validation of the three autorefractors for amblyopia photoscreening.

\section{Spectacle Comparison Ellipsoid Technique}

In our earlier "oval solid" effort, ${ }^{4}$ patients with substantial astigmatism were employed to determine the limits of spherocylinder alteration yielding grade A (1 LogMAR), grade B (3 LogMAR) and grade C (6 LogMAR) blur from optimal, best-corrected visual acuity. The resultant model of concentric ovals on a J0 vs J45 Cartesian was satisfactory for a certain range of refractive error, but misclassified some patients with minimal astigmatism. Therefore, additional subjects with astigmatism ranging from 0.25 to 5.25 diopters were tested. Best retinoscopy with fogging to rule out overaccommodation was then refined in the phoropter yielding the best visual acuity through the "target refraction" which was often 0.25 or more over-minus from maximum hyperopia. The room lights were dim to prevent excess miosis.

From the smallest optotype with horizontal/vertical representation $(\mathrm{H}$ or $\mathrm{T})$, the next larger 1 LogMAR size was projected. Then both spherical and cylinder limits away from target refraction were determined. For sphere, additional spherical lenses were added to determine the threshold above which the optotype could no longer be identified three of four times. For cylinder, additional extra plus cylinder, or spherical equivalent reduced cylinder was added and the axis rotated until the threshold for three of four optotypes above which could not still be identified. If the subject had minimal astigmatism, then with-the-rule and against-the-rule spherical equivalent increasing cylinder was tested until the same threshold was reached. Each set of spherocylinder isospherical equivalent astigmatism were used to define the A-grade astigmatism while increased plus sphere was used to define the A-grade over plus spherical equivalent.

Then the phoropter was returned to target refraction to confirm best acuity and then the chart optotype presented three LogMAR lines larger to represent the B-grade acuity for that subject. Again cylinder was varied in magnitude and axis maintaining spherical equivalent so the threshold limits just below which the B-grade optotypes could no longer be identified three of four times; the set of refractive data constituted the astigmatism spectacles that would blur that subject just 3 LogMAR in a dim room. For spherical equivalent over-plus, from target refraction, 
additional plus sphere was added until that subject could no longer determine three of four of the B-grade optotype.

After returning to target refraction and confirming best visual acuity, then the chart optotype was increased 6 LogMAR to represent the C-grade blur. Combinations of cylinder magnitude and axis were determined to find a set of spectacles just at the $\mathrm{C}$-grade and increased plus sphere added to the target refraction to determine the limit of over-plus for C-grade.

For each A-grade set, each B-grade set and each $\mathrm{C}$-grade set, the astigmatism power and axis were converted to $\mathrm{J} 0$ and $\mathrm{J} 45$ vector transforms.

A-grade is designated for values of 1.00 or less with 0 being identical to the target refraction. The B-grade is for values greater than 1.0 and up to 2.0 while the $\mathrm{C}$-grade is more than 2.0 and up to 3.0. Values over 3.0 are considered a "poor match."

The vector $\mathrm{J} 0$ and $\mathrm{J} 45$ transformations are:

$\mathrm{J} 0=-(\mathrm{Cyl} / 2) * \cos (2 *$ axis $)$

$\mathrm{J} 45=-(\mathrm{Cyl} / 2) * \sin (2 *$ axis $)$

When the subjects with minimal astigmatism were included, the resultant sets of A-grade, B-grade and C-grade refraction astigmatism limits resembled circles with the radius increasing as a function of cylinder power (Cyltarget):

Radius $=$ sqrt $\left((\mathrm{J} 0 \mathrm{t}-\mathrm{J} 0 \mathrm{c})^{2}+(\mathrm{J} 45 \mathrm{t}-\mathrm{J} 45 \mathrm{c})^{2}\right)$

With $\mathrm{t}$ indicating target and $\mathrm{c}$ the candidate refraction, the simplified grade for cylinder related to the target cylinder power $($ Cylt $)=$ radius $/(0.07 *$ Cylt +0.25$)$.

For spherical equivalent $(\mathrm{SphEq}=\mathrm{Spher}+\mathrm{Cyl} / 2)$, The GRADE for over plus grade sphere plus=SphEqc-SphEqt.

For age in years with a maximum of 60 years, the grade for over minus spherical equivalent is related to delta spherical equivalent and age by

Grade sphere minus $=($ SphEqc-SphEqt $) /(0.019 *$ age -1.65$)$.

The ellipsoid combines the $\mathrm{J} 0 \times \mathrm{J} 45$ coordinate plane with a vertical based on spherical equivalent to create a three-dimensional representing the complete spherocylinder (Figure 1). The grade for ellipsoid=sqrt $\left(2 *\right.$ GRADEcyl $^{2}+$ GRADEsph $\left.^{2}\right)$.

The grade for anisometropia is the absolute value of twice the difference in the delta spherical equivalents for right and left eye:

GRADEaniso $=\mid($ SphEqcR-SphEqtR $)-($ SphEqcL SphEqtL) $\mid * 2$.

From the digital spectacle comparison composite, letter grade comparison is defined by A being anything 1 or less, $\mathrm{B}$ up to 2.0 and $\mathrm{C}$ up to 3.0. Anything above 3.0 is defined as a poor fit since that spectacle would not be expected to

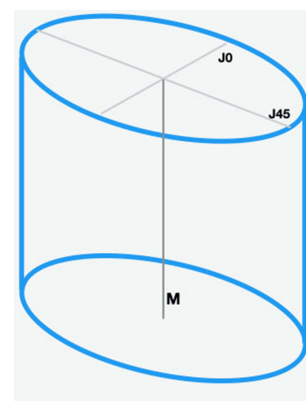

Oval solid

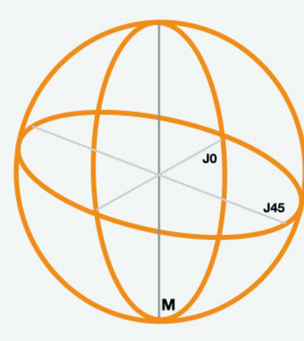

ellipsoid
Figure I Compared oval solid prior $A B C D$ effort with this $A B C D$ composite ellipsoid used to grade spectacle comparison. The horizontal dimension is composed of $\mathrm{J0}$ on $\mathrm{x}$-axis and $\mathrm{J} 45$ on $\mathrm{y}$-axis, while spherical equivalent is on the $\mathrm{z}$-axis with over plus extending up and age-based over-minus tolerance extending down depending on expected accommodation.

allow the patient to resolve better than 6 LogMAR blur from best corrected visual acuity.

Functioning Excel and numbers template databases for these spherocylinder ellipsoid grade matches can be found on the Alaska Blind Child Discovery website (https:// www.abcd-vision.org/vision-screening/Spectacle $\%$ 20Comparison\%20Grade.html).

\section{Comparing Portable Remote Autorefractors Using the Ellipsoid Spherocylinder Grade Technique}

Patients that were consecutive, willing, cooperative and ageappropriate in a pediatric eye practice from January 2020 through February 2021 had photoscreening by experienced screeners with manufacturer-trained protocols and remote autorefraction after accurate retinoscopy. Older patients had refinement at the phoropter after retinoscopy and younger patients had cycloplegic retinoscopy with or without SBARS. ${ }^{5}$ The best visual acuity often was slightly toward myopic from full cycloplegic. Each also had ocular motility, sensorimotor, anterior segment and retinal examination consistent with AAPOS uniform instrument validation. ${ }^{6}$

Each patient was screened with Plusoptix A12 (Nuremberg, Germany) using software version 7.1.5.0. Plusoptix is specifically defined as an infrared, eccentric pediatric photoscreener; it provides binocular spherocylinder refraction estimates from -7 to +5 diopters. Beyond this, it may display "HYP" for excess hyperopia and MYO for excess myopia or it may yield "patient out of focus" for excess refractive interpretations. Plusoptix provides several published options for simple user selection of instrument referral criteria for amblyopia detection ranging from sensitive to specific. ${ }^{7}$ 
Each patient was also screened with the Adaptica 2WIN (Padova, Italy) handheld infrared autorefractor software version V5.0. The 2WIN was handheld and not used with the Kaleidos case. ${ }^{8,9}$ The 2 WIN uses a similar red reflex crescent multiradial method similar to Plusoptix, however, it is primarily designed to yield a wider refractive estimate for adults and children, and therefore, is designed to make spherocylinder estimates up to 7 diopters. The 2 WIN provides white or colored flashing lights and bird-like audio or silent; we mainly used colored flashing lights and bird-like audio to stimulate fixation. The 2 WIN has performed similarly to Retinomax in terms of pediatric refraction. ${ }^{10}$ The 2 WIN can also be directed to monocularly refract one eye, while the other may be patched to allow fixation of a nondominant, strabismic eye.

Each patient was refracted with the Righton Retinomax K-plus 3 (Tokyo, Japan). The Retinomax is held close $(6 \mathrm{~cm})$ in front of one eye for sequential monocular estimation. The patient views an image of a tulip flower in a sunlit field for fixation. The refractionist keeps the device level. Then keratometry and autorefraction by Safir method provides a spherocylinder estimate over a wide range $(-18$ to $+18 \mathrm{D})$ of sphere and cylinder. ${ }^{11}$

\section{Sample Size}

ABCD composite ellipsoid for these autorefractors has a mean between one and two with a standard deviation of about one. To detect a difference of 0.2 from a mean of 2.0 with standard deviation 1.0 , for alpha of 0.05 and power 0.8 , a sample size of 393 is needed.

\section{Spectacle Need}

Patients with a refraction near plano sphere with minimal cylinder have minimal need of spectacles for distance viewing. Alternatively, patients with a high sphere over five diopters and/or cylinder over four diopters have a high need for spectacle correction. We, therefore, devised a set of criteria ranking the level of spectacle need illustrated in Figure 2. Patients with actual refraction spherical equivalent of five or more diopters, and/or cylinder of four or more diopters are the highest need defined as level 3 . Level 2 moderately high need has lower limits of spherical equivalent 3.5 diopters and lower limit of astigmatism at two diopters. Mild spectacle need grade one has lower limit of myopia at one diopter, hyperopia at two diopters and cylinder at one diopter. Minimal spectacle need level 0 have spherical equivalent and cylinder levels less than one diopter myopia, less than two diopters hyperopia and/or less than one diopter cylinder.

\section{Validation of Noncycloplegic Autorefractions for Amblyopia Risk Factor Screening}

Using AAPOS 2013 Uniform Guidelines ${ }^{6}$ and the older of the three age triads, the Plusoptix, the Retinomax and the 2WIN were compared with idealized sensitive, specific and mid-range instrument referral criteria to generate a receiver operating characteristic (ROC) curve. ${ }^{12}$ Additional calculations were made using the proposed 2021 AAPOS uniform guidelines. ${ }^{13}$ Inconclusive and outof-range interpretations were considered as a referral.

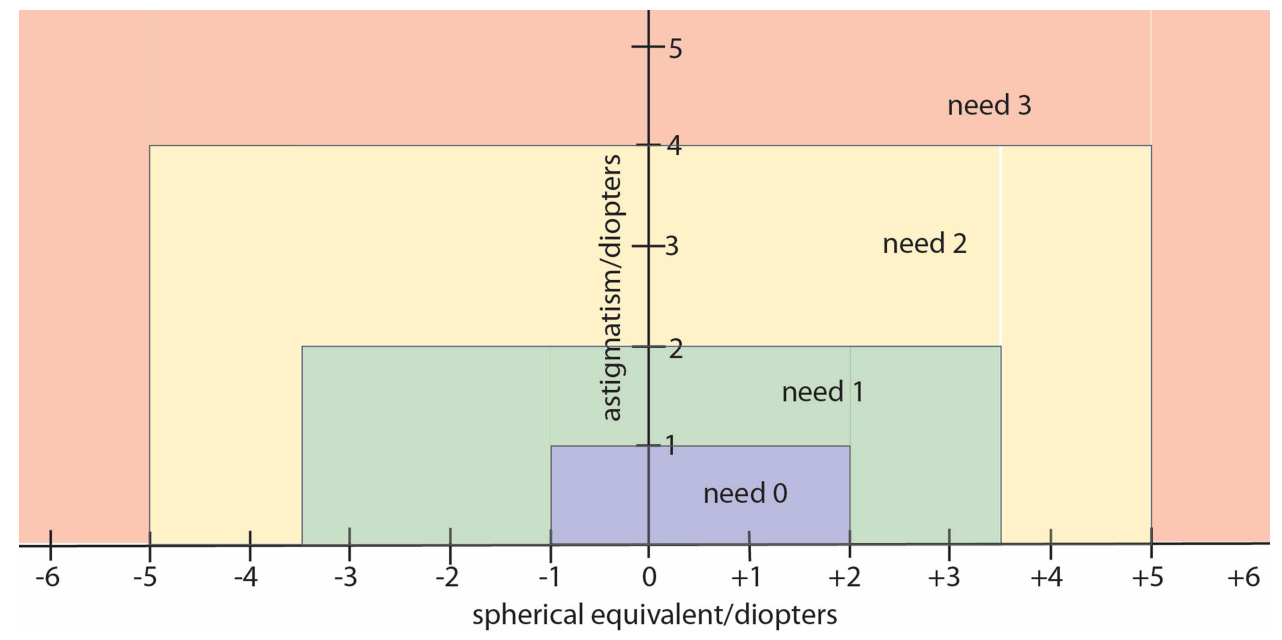

Figure 2 Limits of spectacle refraction sphere and cylinder spectacle components that indicate the low or high level optical need relative to resolving blur. 


\section{Results}

A total of 202 patients with confirmatory exams were screened by all three portable autorefracting devices. Ages ranged from 1 to 19 years with mean $7.4 \pm 4$ years and median 6.3 years. There were 103 females. Racial/ ethnic breakdown was 14 Asian, 18 Black, 30 Hispanic, 9 Alaska Native, 16 Pacific Islanders and 115 White. Pediatrician or community photoscreening referred 32 while new referral of visual acuity and other reasons were 24; the remaining were follow-up exams of which 64 had a diagnosis of amblyopia and 39 had a diagnosis of constant or intermittent strabismus. Thirty-five had a diagnosis of developmental delay including four with autism, 11 with attention deficit and five with marked prematurity. De-identified data for this comparative study can be retrieved from: https://www.abcd-vision.org/refer ences/ABCD\%20composite\%20raw.pdf
From the confirmatory exam refractions, 33 eyes had high level 3 spectacle need, 81 eyes had moderately high level 2 spectacle need, 163 had moderate level 1 spectacle need and 127 had low level 0 spectacle need. Spherical equivalent $\mathrm{M}$ ranged from $-7 \mathrm{D}$ to $+7 \mathrm{D}, \mathrm{J} 0$ ranged from -1.00 to 2.83 and $\mathrm{J} 45$ ranged from -0.98 to 1.08 .

Unreadable or inconclusive results were three patients/ six eyes for $2 \mathrm{WIN}$, six patients/12 eyes for Retinomax (mainly young children) and 18 patients/23 eyes for Plusoptix usually due to the patient being out of range.

Table 1 gives intraclass correlation (ICC), Bland-Altman metrics, and $\mathrm{ABCD}$ composite results when Plusoptix, Retinomax, and 2WIN were compared to confirmatory exam for all patients and for eyes with higher spectacle need (combined level 2 and level 3 spectacle need). Figure 3 shows Bland-Altman graphs for spherical

Table I Spectacle Comparison

\begin{tabular}{|c|c|c|c|c|c|c|c|c|}
\hline Exam vs & & & Ellipsoid & Sphere/M & Cyl & J0 & J45 & Aniso \\
\hline \multirow[t]{8}{*}{ PlusoptiX } & ALL & ICC & & 0.72 & & 0.88 & 0.57 & \\
\hline & & BA mean difference & & 0.39 & & -0.07 & 0.00 & \\
\hline & & BA SD difference & & 1.15 & & 0.33 & 0.21 & \\
\hline & & ABCD composite & 1.68 & 0.74 & 0.86 & & & 0.75 \\
\hline & Need 2 and 3 & ICC & & 0.65 & & 0.84 & 0.66 & \\
\hline & & BA mean difference & & 0.99 & & 0.03 & 0.01 & \\
\hline & & BA SD difference & & 1.62 & & 0.43 & 0.31 & \\
\hline & & $A B C D$ composite & 2.03 & 1.00 & 0.91 & & & 0.75 \\
\hline \multirow[t]{8}{*}{ Retinomax } & ALL & ICC & & 0.57 & & 0.90 & 0.59 & \\
\hline & & BA mean difference & & 1.55 & & 0.02 & 0.00 & \\
\hline & & BA SD difference & & 1.77 & & 0.27 & 0.21 & \\
\hline & & $A B C D$ composite & 1.55 & 0.77 & 0.72 & & & 1.25 \\
\hline & Need 2 and 3 & ICC & & 0.66 & & 0.91 & 0.69 & \\
\hline & & BA mean difference & & 1.54 & & 0.00 & -0.01 & \\
\hline & & BA SD difference & & 1.85 & & 0.28 & 0.22 & \\
\hline & & ABCD composite & 1.55 & 0.75 & 0.8 & & & 1.50 \\
\hline \multirow[t]{8}{*}{$2 \mathrm{WIN}$} & ALL & ICC & & 0.67 & & 0.80 & 0.49 & \\
\hline & & BA mean difference & & 0.78 & & -0.14 & 0.05 & \\
\hline & & BA SD difference & & 1.30 & & 0.39 & 0.26 & \\
\hline & & ABCD composite & 1.72 & 0.56 & 1.00 & & & 0.50 \\
\hline & Need 2 and 3 & ICC & & 0.65 & & 0.88 & 0.58 & \\
\hline & & BA mean difference & & 1.60 & & -0.08 & 0.07 & \\
\hline & & BA SD difference & & 1.93 & & 0.45 & 0.34 & \\
\hline & & ABCD composite & 2.44 & 1.13 & 1.14 & & & 1.00 \\
\hline
\end{tabular}

Notes: Degree of spherocylinder similarity between refined retinoscopy and three portable autorefractors, Plusoptix AI2, Retinomax K Plus and Adaptica WIN. The $A B C D$ composite is a method devised by the Alaska Blind Child Discovery that uses limits of spectacle blur to create an oval from J0 and J45 components into Cyl, an ageaccommodation based sphere for over and under spherical equivalent, and finally a three-dimensional ellipsoid which combines Cyl and sphere.

Abbreviations: ALL, 202 children less than 20 years old; Need 2 and 3, the 113 eyes with higher level spectacle requirements; ICC, intraclass correlation; BA, BlandAltman; SD, standard deviation; M, spherical equivalent; Aniso, anisometropia; J0 and J45, vector transformations for astigmatism. 


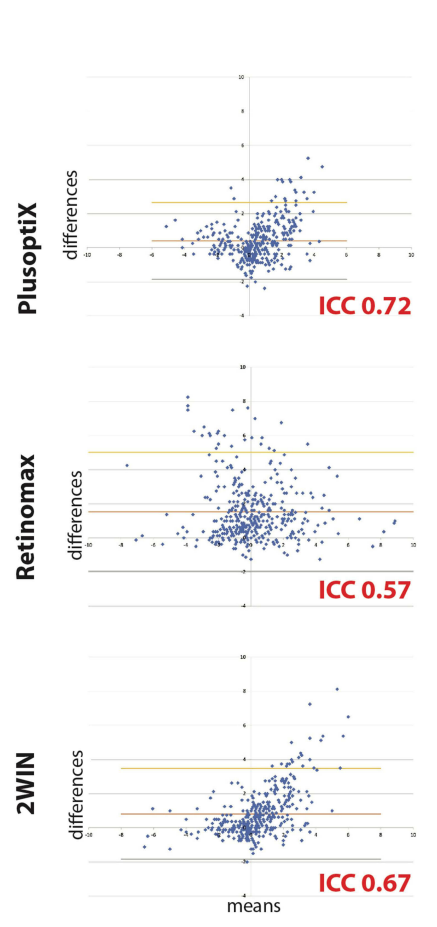

Spherical Equivalent $(M)$

\section{Bland-Altman}

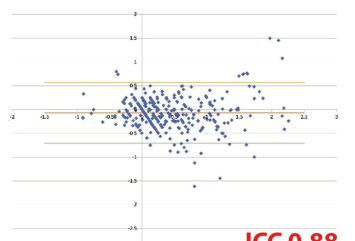

ICC 0.88

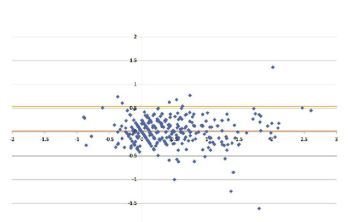

ICC 0.90

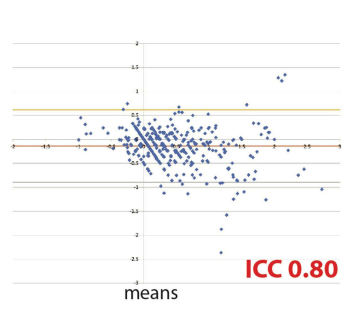

J0 Vector

\section{Ellipsoid}
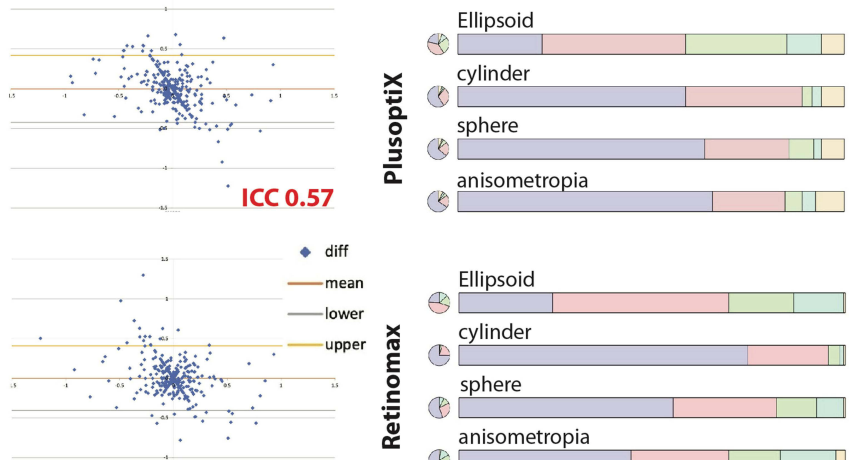

ICC 0.59

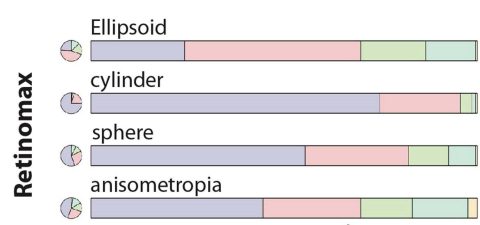

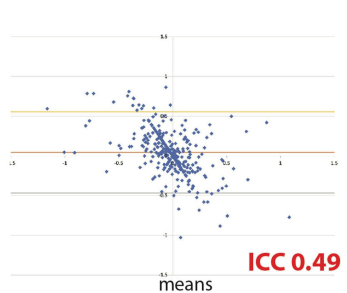

J45 Vector

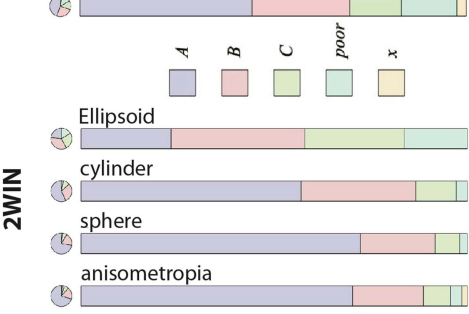

Spectacle Compare "GRADE"

Figure 3 Bland-Altman and ABCD composite grade for three handheld autorefractors comparing spectacle components spherical equivalent, $J 0$ and $\mathrm{J} 45$ cylinder vector transformations for all 202 pediatric patients with high and low spectacle needs.

\section{Bland-Altman}
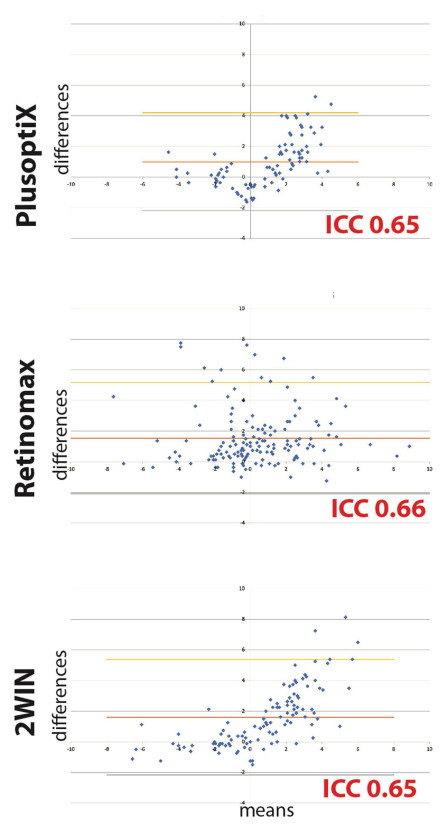

Spherical Equivalent (M)
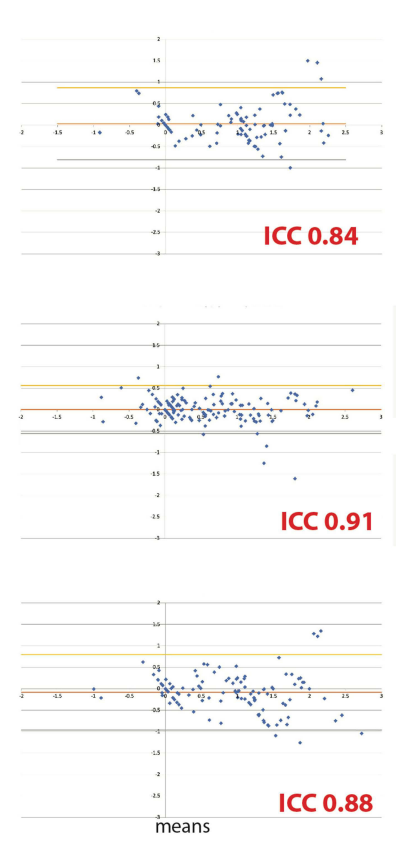

J0 Vector
High Spectacle Need $(2 \& 3)$

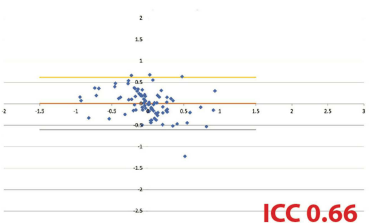

ICC 0.66

ICC 0.69

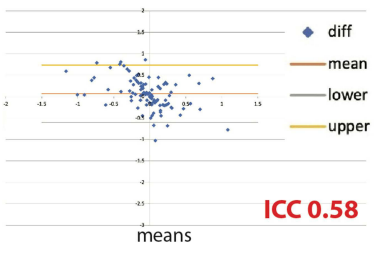

J45 Vector
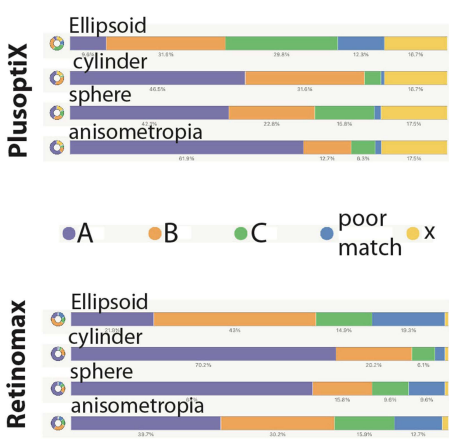

\section{Ellipsoid}

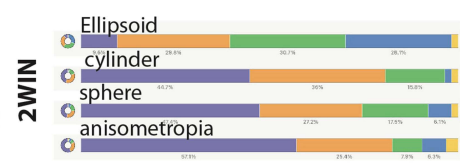

Spectacle Compare "GRADE"

Figure 4 Bland-Altman and ABCD composite comparison for the three handheld remote autorefractors for the $\mathrm{n}=113$ eyes from pediatric patients with higher level 2 and level 3 spectacle needs. 
equivalent, J0 and J45 vector transformation when Plusoptix, Retinomax and 2 WIN are compared with confirmatory exam for all patients with direct comparison with bar graphs of ABCD composite outcomes for anisometropia, cylinder, spherical equivalent and overall ellipsoid. Figure 4 resembles Figure 3 but is limited to the 113 eyes with higher level 2 and 3 spectacle need.

For all patients, with ABCD composite achieving a grade of $\mathrm{B}$ or higher, Plusoptix had spherical equivalent $86 \%$, cylinder $89 \%$ and ellipsoid $62 \%$. Retinomax had $90 \%$ spherical equivalent, 95\% cylinder and $68 \%$ ellipsoid while 2 WIN had spherical equivalent $75 \%$, cylinder $85 \%$ and ellipsoid 58\%. Even with high level 2 and 3 spectacle need, earning a grade of B or higher, Plusoptix had spherical equivalent $65 \%$, cylinder $78 \%$ and ellipsoid $41 \%$. Retinomax had 80\% spherical equivalent, $90 \%$ cylinder and $65 \%$ ellipsoid while 2 WIN had spherical equivalent $75 \%$, cylinder $85 \%$ and ellipsoid $39 \%$.

Utilizing the $\mathrm{ABCD}$ composite, there was a significant difference between the handheld autorefractors in estimating confirmatory refraction with anisometropia worse for sequential monocular Retinomax (Kruskal-Wallis $\mathrm{X}^{2}(2)$ $=41, p<0.001$ ), better for spherical equivalent with $2 \mathrm{WIN}$ (Kruskal-Wallis $\mathrm{X}^{2}(2)=19, p<0.001$ ), better for composite cylinder with Retinomax (Kruskal-Wallis $X^{2}(2)=45$, $p<0.001)$ and slightly better composite ellipse with Retinomax (Kruskal-Wallis $\quad \mathrm{X}^{2}(2)=7.1, \quad p=0.03$ ) Comparing just the two infrared photoscreeners, median anisometropia for Plusoptix 0.75 diopters did not differ from 0.5 diopters for $2 \mathrm{WIN}$ (Mann-Whitney $\mathrm{z}=0.66$, $p=0.51$ ), the median spherical equivalent Plusoptix of +0.74 diopters did not differ from +0.56 diopters for 2WIN (Mann-Whitney 1.77, $p=0.08$ ), median composite $\mathrm{J} 0 \times \mathrm{J} 45$ cylinder Plusoptix 0.86 was less than 1.00 for 2WIN (Mann-Whitney 2.8, $p=0.005$ ), but ellipsoid for Plusoptix 1.68 did not differ from 1.72 for $2 \mathrm{WIN}$ (Mann-Whitney 1.48, $p=0.14$ ).

Figure 5 shows correlations of each continuous variable from the ABCD composite spherical equivalent, $\mathrm{J} 0 \times \mathrm{J} 45$ cylinder and ellipsoid comparing each handheld remote refracting device. A high correlation was shown for most interactions but not for spherical equivalent especially for Retinomax. Spherical equivalent Plusoptix correlated with 2 WIN (Spielman $\mathrm{r}=0.55, p<0.001$ ).

The grade match worsens with increasing spectacle need for Plusoptix (Kruskal Wallis $\mathrm{X}^{2}(3)=29, p<0.001$ ) and 2WIN (Kruskal-Wallis $X^{2}(3)=34, p<0.01$ ) but not for Retinomax (Kruskal-Wallis $\mathrm{X}^{2}(3)=2.4, p=0.49$ ).
The ellipsoid was negatively correlated with age for Retinomax (product moment $\mathrm{r}(396)=-0.23, p<0.001$ ) both due to cylinder $(p=0.008)$ and even more for spherical equivalent $(\mathrm{r}(395)=-0.22, p<0.001)$, but not ellipsoid for Plusoptix $(\mathrm{r}(380)=-0.06, p=0.26)$ and $2 \mathrm{WIN}(\mathrm{r}(399)=$ $-0.03, p=0.50)$. Spherical equivalent was mildly negative correlated for Plusoptix $(\mathrm{r}(380)=-0.12, p=0.02)$ but not for $2 \mathrm{WIN}$.

Compared to AAPOS confirmatory exam, using the older triad of the 2013 Uniform guidelines (Gold Standard Hyperopia $>3.5 \mathrm{D}$, cylinder $>1.5 \mathrm{D}$, myopia more than 1.5 D and anisometropia more than $1.5 \mathrm{D})$, and instrument referral criteria shown in Table 2, sensitivity/specificity/PPV for Plusoptix were $80 \% / 83 \% / 79 \%$, for 2WIN $72 \% / 88 \% / 82 \%$ and for Retinomax 84\%/73\%/71\%. Additional sensitive and specific instrument referral criteria for 2013 guidelines and the proposed 2021 guidelines are shown in Figure 6.

\section{Discussion}

A simplified method for spectacle comparison based on visual blur has been developed. The ABCD ellipsoid grade closely resembles the more complex Bland-Altman analysis for three handheld autorefractors in children. The manifest Safir-method Retinomax autorefractor compared to the infrared multiradial photoscreeners Plusoptix and 2WIN better estimated the nuances of astigmatism especially in high-need refractions, but less well estimated cycloplegic hyperopia.

Retinomax refraction estimate was better in older children for sphere, cylinder and ellipsoid- more so than Plusoptix and 2WIN. This was particularly true of sphere for which the near impression of the target in Retinomax seemed to stimulate accommodation in the younger children.

For patients with high spectacle need, who presumably would fail an unaided distance visual acuity test, an ABCD composite ellipsoid comparison grade autorefraction of A or B expected to yield $20 / 40$ or better was shown by $41 \%$ of Plusoptix, $39 \%$ of 2 WIN and $65 \%$ by Retinomax. All three would provide a reasonable estimate of refraction in a remote location, however the price difference from $\$ 6000-7000$ for the infrared photoscreeners to $\$ 13,000$ for the Retinomax should guide and inform the selection for a given clinic.

The performance in amblyopia risk factor determination was good for all three non-cycloplegic handheld autorefractors, but Retinomax tended to under-read hyperopia 


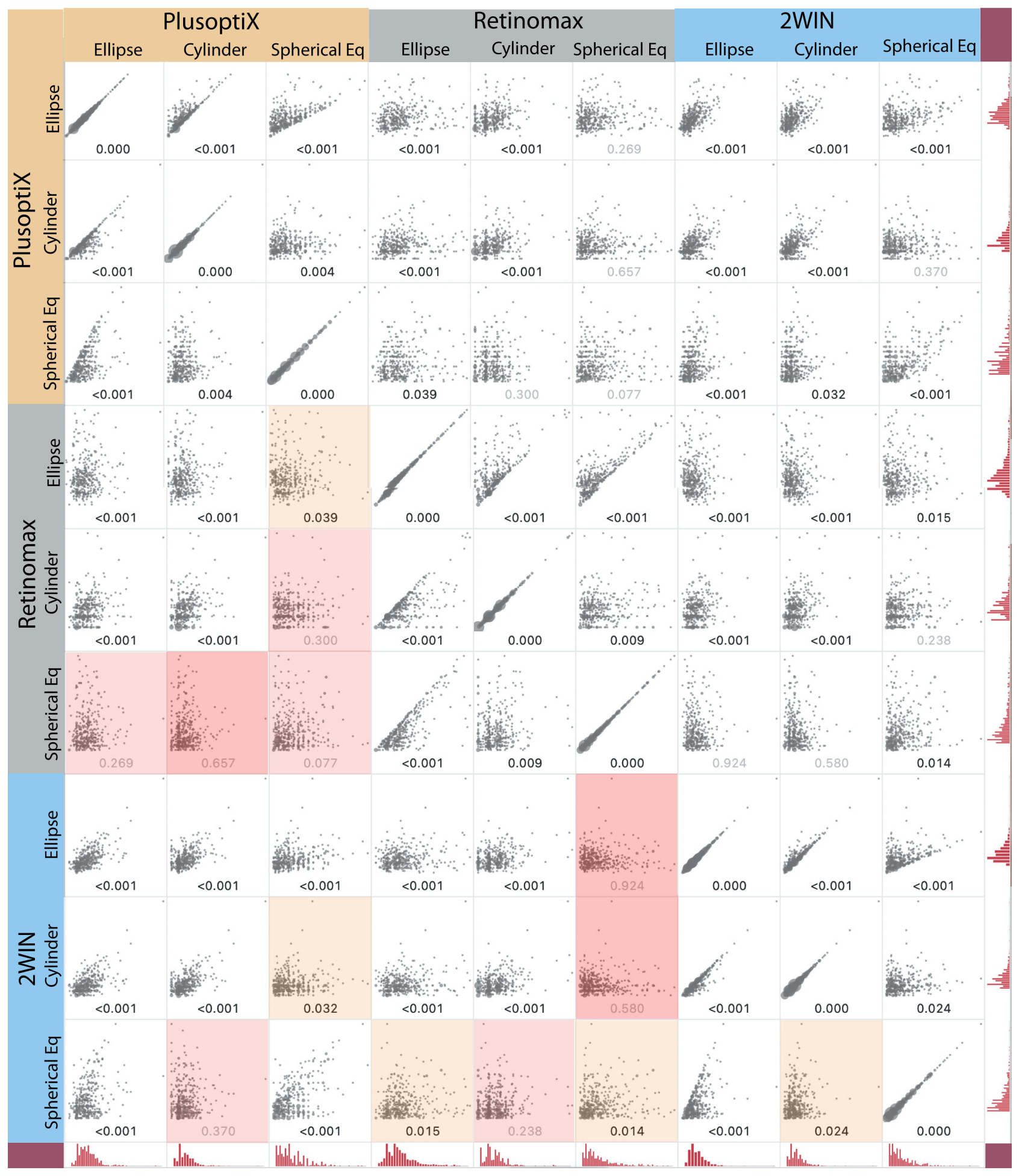

Figure 5 A comparison of correlations comparing three ABCD composite components (spherical equivalent, composite j0×J45 cylinder and ellipsoid) for the Plusoptix, 2WIN and Retinomax handheld autorefractors. 
Table 2 Validation Statistic for Amblyopia Screening

\begin{tabular}{|c|c|c|c|c|c|c|c|c|c|c|}
\hline \multicolumn{2}{|c|}{ AAPOS Amblyopia Detection } & & Instrument Referral Criteria & Sensitivity & Specificity & PPV & A & B & C & D \\
\hline \multirow[t]{6}{*}{ Plusoptix } & \multirow[t]{3}{*}{2013} & Sens & $\mathrm{H} 2 \mathrm{Cl} .5 \mathrm{M} 3 \mathrm{Al} .25$ & $91 \%$ & $72 \%$ & $72 \%$ & 81 & 32 & 8 & 81 \\
\hline & & Refer & H2.5 C2 M3.5 Al. 5 & $80 \%$ & $83 \%$ & $79 \%$ & 71 & 19 & 18 & 94 \\
\hline & & Spec & H3 C2.5 M4 A2 & $67 \%$ & $94 \%$ & $90 \%$ & 60 & 7 & 29 & 106 \\
\hline & \multirow[t]{3}{*}{2021} & Sens & $\mathrm{M} 2 \mathrm{H} 2.25 \mathrm{Cl} .75 \mathrm{Al} .25$ & $95 \%$ & $76 \%$ & $75 \%$ & 83 & 28 & 4 & 87 \\
\hline & & Refer & M2.5 H2.5 C2 Al.5 & $83 \%$ & $83 \%$ & $78 \%$ & 72 & 20 & 15 & 95 \\
\hline & & Spec & M2.5 H3.5 C2.5 Al.75 & $64 \%$ & $94 \%$ & $89 \%$ & 56 & 7 & 31 & 108 \\
\hline \multirow[t]{6}{*}{ Retinomax } & \multirow[t]{3}{*}{2013} & Sens & H2.5 Cl.25 M3 Al.I & $96 \%$ & $58 \%$ & $64 \%$ & 85 & 48 & 4 & 65 \\
\hline & & Refer & H3 Cl.75 M3.25 Al.75 & $84 \%$ & $73 \%$ & $71 \%$ & 75 & 30 & 14 & 83 \\
\hline & & Spec & H3.5 C2 M3.5 A2 & $78 \%$ & $94 \%$ & $91 \%$ & 69 & 7 & 20 & 106 \\
\hline & \multirow[t]{3}{*}{2021} & Sens & H3 Cl.5 MI.75 Al.25 & $93 \%$ & $48 \%$ & $57 \%$ & 81 & 60 & 6 & 55 \\
\hline & & Refer & H3.5 Cl.75 M2 Al.5 & $90 \%$ & $58 \%$ & $62 \%$ & 78 & 48 & 9 & 67 \\
\hline & & Spec & H4 C2 M2.5 A2 & $80 \%$ & $72 \%$ & $69 \%$ & 70 & 32 & 17 & 83 \\
\hline \multirow[t]{6}{*}{$2 \mathrm{WIN}$} & \multirow[t]{3}{*}{2013} & Sens & HI.5 Cl.75 M3 Al.5 & $90 \%$ & $70 \%$ & $70 \%$ & 80 & 34 & 9 & 79 \\
\hline & & Refer & H2.5 C2.25 M3.25 Al.75 & $72 \%$ & $88 \%$ & $82 \%$ & 64 & 14 & 25 & 99 \\
\hline & & Spec & H3 C2.5 M3.5 A2 & $58 \%$ & $91 \%$ & $84 \%$ & 52 & 10 & 37 & 103 \\
\hline & \multirow[t]{3}{*}{2021} & Sens & HI.75 CI.5 MI.75 AI.25 & $92 \%$ & $63 \%$ & $65 \%$ & 80 & 43 & 7 & 72 \\
\hline & & Refer & H2.25 C2 M2 Al. 5 & $87 \%$ & $81 \%$ & $78 \%$ & 76 & 22 & II & 93 \\
\hline & & Spec & H2.75 C2.25 M2.5 A1.75 & $75 \%$ & $88 \%$ & $82 \%$ & 65 & 14 & 22 & 101 \\
\hline
\end{tabular}

Notes: Three non-cycloplegic portable autorefractors, the Plusoptix AI2, The Retinomax, and the Adaptica 2 WIN, are compared to AAPOS 2013 older triad guidelines ${ }^{6}$ and proposed 2021 guidelines. Instrument referral criteria for each device are customized to offer a more sensitive (Sens) option, a more specific option (SpeC), and a routine (Refer) option. The criteria are all those interpretations with hyperopia $(H)$, cylinder $(C)$, myopia $(M)$ and anisometropia $(A)$ greater than or equal to the adjacent number in diopters.

Abbreviations: PPV, positive predictive value; A, number referred with risk factor; B, number referred without risk factors; C, number not referred but with risk factors; $\mathrm{D}$, number not referred without risk factors.

and overestimate myopia presumably by inducing more accommodation.

The performance in refractive estimate was not dependent on spectacle need for Retinomax, but was less reliable with increasing spectacle need for Plusoptix (the device that does not give an answer above 7 diopters) and 2WIN.

The 2WIN and the Plusoptix A12R were recently compared in 142 young children ${ }^{14}$ with $5 \%$ inconclusive with 2 WIN and $11 \%$ with Plusoptix. The 2 WIN was designed to estimate refractive error in adults and children whereas Plusoptix limits the range of refractive estimates designating high values as "refer" for amblyopia screening. Plusoptix had sensitivity/specificity of 73\%/96\% compared to $67 \% / 84 \%$ for 2 WIN. Both underestimated compared to cycloplegic hyperopia, but overestimated astigmatism. In another study of 100 children, Plusoptix and $2 \mathrm{WIN}$ were better at estimating sphere than cylinder compared to Topcon KR-8900 autorefractor. ${ }^{15}$

Cycloplegic Retinomax in younger children lagged behind retinoscopy with respect to uncovering hyperopia especially in younger children. ${ }^{16}$ The dry Grand Seiko was slightly better than the dry Topcon KR800 at detecting cycloplegic refractive error in 886 Chinese school children. ${ }^{17}$ The dry Retinomax did a reasonable job compared to table-top Nikon NRK 8000 autorefractor in children mean age 97 months. ${ }^{18}$ Dry Retinomax retinoscopy in 1218 children was more reliable for astigmatism than for anisometropia. $^{19}$ An earlier software version of the Plusoptix was similarly sensitive, but less specific for detection of higher levels of astigmatism than the SPOT in 105 children. $^{20}$

Four separate experienced retinoscopists in 117 patients 5-60 years old were recently compared to three different autorefractive methods: the rotary prisms Topcon RM 8900 , the multiradial eccentric infrared photoscreening in the SPOT and the wavefront aberrometry in the E-see. ${ }^{21}$ Nine subjects had inconclusive SPOT and were excluded. Bland-Altman analysis showed similar inter-equipment $\mathrm{M}$ and J0 bias that was not age dependent. Reliability for repeat testing was good at $0.75 \mathrm{D}$ for $\mathrm{M}$ and 0.40 for $\mathrm{J} 0$. There were relatively large agreement limits of $\pm 2.5 \mathrm{D}$ for $\mathrm{M}$ and $\pm 1 \mathrm{D}$ for $\mathrm{J} 0$ with the wavefront performing a bit better.

Strengths of this study are ample sample size of children from diverse ethnic and developmental backgrounds and 


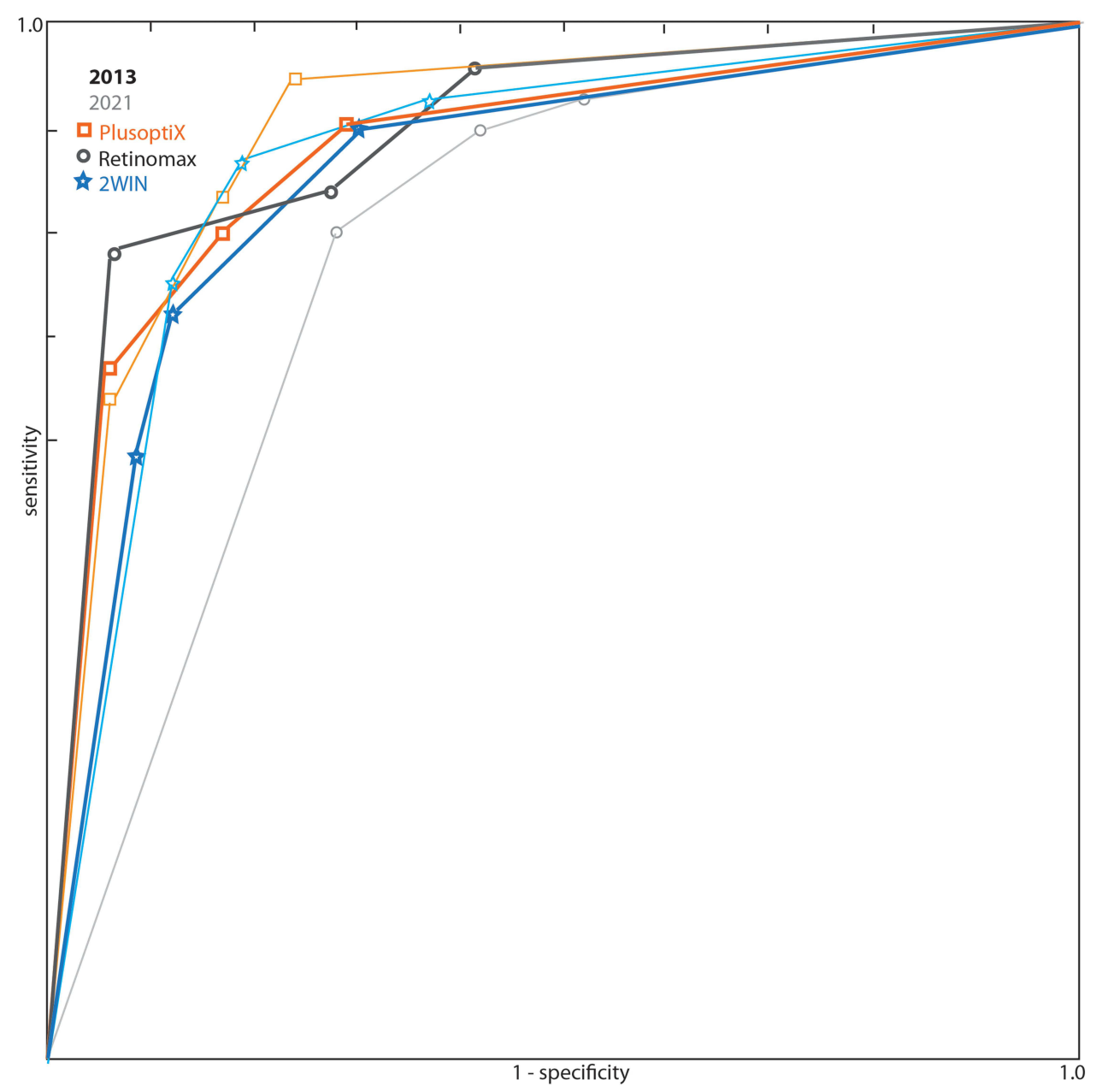

Figure 6 Receiver operating characteristic (ROC) curve comparing portable autorefractors Plusoptix SI2 (orange squares), Adaptica 2 WIN (blue stars) and Retinomax (gray circles) for 202 pediatric patients with high $43 \%$ prevalence refractive amblyopia risk factors. The older triad of the 2013 AAPOS uniform guideline is shown in bold compared to the proposed 2021 AAPOS Uniform Guidelines for patients older than four years shown in delicate lines.

covering a wide range of refractive error. Three state-of-theart, updated handheld autorefractors are simultaneously compared in over 200 patients. The study employs uniform standards of intraclass correlation, Bland-Altman and also AAPOS amblyopia risk factor comparison. Weaknesses of the study include not utilizing different accommodation fixation stimuli lights or targets for $2 \mathrm{WIN}$ and Retinomax. Also, to achieve adequate sample size, both right and left eyes of the 202 subjects were included recognizing that for the nonanisometropic patients, spherocylinder of the right eye often mirrors the left. Not all of the older patients used cycloplegia as the method to relax their accommodation. The median age of patients was over 7 years and considered relatively late for amblyopia screening.

Simplified spectacle comparison might be helpful in the following scenarios. First, as in this paper, non-eye doctors and lay screening clinics comparing autorefractors designed to screen children for amblyopia is a practical example. Second, the resultant refractions for a novice refractionist could be quickly compared based on resultant blur and graded with an expert teacher. The third example is the collection and dispensing of donated spherocylinder spectacles in remote regions of the world for which affordable optical stores are limited; American charitable spectacle centers such as Lion's clubs often discard donated cylinder spectacles since there has been no simple and practical way for non-eye doctors to provide remote dispensing. An optically naïve volunteer with a remote autorefractor, a catalogued collection of donated spherocylinder spectacles and a smart phone with $\mathrm{ABCD}$ composite ellipsoid grading might reduce blindness where optical shops are too sparse. This paper does not yet provide evidence for some of these potential applications. Whether this refraction comparison will aid in practical dispensing of donated spherocylinder 
spectacles will require in-the-field observation of the non-eye specialists at task.

Astigmatism is an important and complex concept related to refractive amblyopia and vision impairment due to uncorrected refractive error in remote parts of the world. Since $2003,{ }^{22}$ the magnitude, but not the axis of astigmatism is included in the AAPOS refractive amblyopia risk factors. ${ }^{6,13}$ Moreover, axis is an important aspect contributing to reduction of blur and tolerance of newly provided spectacles. Vector transformation is the gold standard for analysis of astigmatism. $^{23}$ A simple A-grade, B-grade or C-grade blur resolution match between a target and a candidate spectacle should be much simpler for optically naïve individuals involved in addressing uncorrected refractive error worldwide.

\section{Funding}

There is no funding to report.

\section{Disclosure}

Robert Arnold is board member of Glacier Medical Software that makes cloud-based ROP Check, is board member of PDI Check that makes vision screening games for Nintendo 3DS autostereoscopic screen, is coordinator of the Alaska Blind Child Discovery that has received discounted vision screening technology from several vendors, and is investigator and protocol developer for NIHsupported Pediatric Eye Disease Investigator Group (PEDIG). In addition, he is a member of the non-paid advisory boards for Plusoptix, GoCheck Kids, Adaptica, and iScreen. Kyle Smith is board member of PDI Check. The authors report no other conflicts of interest in this work.

\section{References}

1. Pearce MG. Clinical outcomes following the dispensing of ready-made and recycled spectacles: a systematic literature review. Clin Exp Optom. 2014;97(3):225-233. doi:10.1111/cxo.12126

2. Cotter SA, Edwards AR; Pediatric Eye Disease Investigator Group. Treatment of anisometropic amblyopia in children with refractive correction. Ophthalmology. 2006;113(6):895-903. doi:10.1016/j. ophtha.2006.01.068

3. Miller JM. Clinical applications of power vectors. Optom Vis Sci. 2009;86(6):599-602. doi:10.1097/OPX.0b013e3181a6a211

4. Beveridge J, Martin SJ, Beveridge N, Metzger EA, Smith KA, Arnold RW. Grading spectacle similarity. Clinical Optometry. 2021;13:23-32. doi:10.2147/OPTO.S289770

5. Arnold AW, Arnold SL, Sprano JH, Arnold RW. School bus accommodation-relaxing skiascopy. Clin Ophthalmol. 2019;13:1841-1851. doi:10.2147/OPTH.S219031

6. Donahue SP, Arthur B, Neely DE, Arnold RW, Silbert D, Ruben JB. Guidelines for automated preschool vision screening: a 10-year, evidence-based update. $J$ AAPOS. 2013;17(1):4-8. doi:10.1016/j. jaapos.2012.09.012
7. Singman E, Matta N, Tian J, Silbert D. A comparison of referral criteria used by the plusoptiX photoscreener. Strabismus. 2013;21 (3):190-194. doi:10.3109/09273972.2013.811606

8. Keffalos MA, Martin SJ, Arnold RW. Drive-by photoscreening: plusoptiX, 2WIN and blinq amblyopia detection during the COVID-19 pandemic. Clin Ophthalmol. 2021;15:775-782. doi:10.2147/OPTH.S300871

9. Martin SJ, Htoo HE, Hser N, Arnold RW. Performance of two photoscreeners enhanced by protective containers. Clin Ophthalmol. 2020;14:1427-1435. doi:10.2147/OPTH.S251451

10. Levitt AH, Martin SJ, Arnold RW. Performance of glow-fixation GCK and 2WIN photoscreeners and retinomax to uncover hyperopia. Clin Ophthalmol. 2020;14:2237-2244. doi:10.2147/ OPTH.S256991

11. Safir A, Kulikowski C, Deuschle K. Automatic refraction: how it is done: some clinical results. Sight Sav Rev. 1973;43(3):137-148.

12. Miller JM. Designing a rational screening program. Am Orthopt J. 2006;56(1):30-34. doi:10.3368/aoj.56.1.30

13. Arnold RW, Donahue SP, Silbert DI, et al. Uniform guidelines for pediatric vision screen validation 2021. J AAPOS. In press 2021.

14. Racano E, Alessi S, Pertile R. Comparison of 2Win and plusoptiX A12R refractometers with Retinomax handheld autorefractor keratometer. $J$ AAPOS. 2019;23(5):276e1-276 e5. doi:10.1016/j. jaapos.2019.05.017

15. Yalcin E, Sultan P, Yilmaz S, Pallikaris IG. A comparison of refraction defects in childhood measured using plusoptix S09, 2WIN photorefractometer, benchtop autorefractometer, and cycloplegic retinoscopy. Semin Ophthalmol. 2017;32(4):422-427. doi:10.3109/ 08820538.2015.1118135

16. Gole GA, Schluter PJ, Hall J, Colville D. Comparison of the Retinomax autorefractor with hand-held retinoscopy in 1-year-old infants. Clin Exp Ophthalmol. 2003;31(4):341-347. doi:10.1046/ j.1442-9071.2003.00668.x

17. Wang D, Jin N, Pei RX, et al. Comparison between two autorefractor performances in large scale vision screening in Chinese school age children. Int $J$ Ophthalmol. 2020;13(10):1660-1666. doi:10.18240/ ijo.2020.10.22

18. Tuncer I, Zengin MO, Karahan E. Comparison of the Retinomax hand-held autorefractor versus table-top autorefractor and retinoscopy. Int J Ophthalmol. 2014;7(3):491-495.

19. Cordonnier M, Kallay O. Non-cycloplegic screening for refractive errors in children with the hand-held autorefractor Retinomax: final results and comparison with non-cycloplegic photoscreening. Strabismus. 2001;9(2):59-70. doi:10.1076/stra.9.2.59.701

20. Crescioni M, Miller JM, Harvey EM. Accuracy of the spot and plusoptix photoscreeners for detection of astigmatism. $J$ AAPOS. 2015;19(5):435-440. doi:10.1016/j.jaapos.2015.07.284

21. Padhy D, Bharadwaj SR, Nayak S, Rath S, Das T. Does the accuracy and repeatability of refractive error estimates depend on the measurement principle of autorefractors? Transl Vis Sci Technol. 2021;10 (1):2. doi:10.1167/tvst.10.1.2

22. Donahue S, Arnold R, Ruben JB. Preschool vision screening: what should we be detecting and how should we report it? Uniform guidelines for reporting results from studies of preschool vision screening. J AAPOS. 2003;7(5):314-316. doi:10.1016/S1091-8531(03)00182-4

23. Thibos LN, Wheeler W, Horner D. Power vectors: an application of Fourier analysis to the description and statistical analysis of refractive error. Optom Vis Sci. 1997;74(6):367-375. doi:10.1097/00006324199706000-00019 


\section{Publish your work in this journal}

Clinical Ophthalmology is an international, peer-reviewed journal covering all subspecialties within ophthalmology. Key topics include: Optometry; Visual science; Pharmacology and drug therapy in eye diseases; Basic Sciences; Primary and Secondary eye care; Patient Safety and Quality of Care Improvements. This journal is indexed on PubMed
Central and CAS, and is the official journal of The Society of Clinical Ophthalmology (SCO). The manuscript management system is completely online and includes a very quick and fair peer-review system, which is all easy to use. Visit http://www.dovepress.com/ testimonials.php to read real quotes from published authors. 